\title{
TWENTY-SEYENTH ANNUAL SASKATCHEWAN CHRISTMAS BIRD COUNT, 1968
}

\section{Compiled by Mary Houston, 863 University Drive, Saskatoon}

In spite of very bitter weather during the 1968 Christmas Count period enthusiastic bird counters throughout the province turned out in force. Thirty-eight counts were sent in from Saskatchewan localities, surpassing 1960's high of 34 . In addition one count was received from Alberta and one from the Northwest Territories. Fifty-two counters turned out in Saskatoon to compile the largest number of species, 36 , ever counted in their area on a Christmas count.

As usual Black-billed Magpies (in 36 localities) and House Sparrows (in 33) were the most frequently recorded species. Bohemian Waxwings were widespread - being counted in 28 areas. They were followed closely by Black-capped Chickadees (28), Common Redpolls (28) and Snow Buntings (26).

One new species was added to the all-time total - a Red-necked Grebe, reported from Regina. In additional species seen, another first is provided for the provincial list of Christmas count species by the Oldsquaw taken in the Fort Qu'Appelle area on December 21, 1968. The duck was a female, in between summer and winter plumage, taken at Sioux Bridge at the east end of Pasqua Lake on an open stretch of water. Jack Lowe and his son Jay were later able to acquire the specimen for E. Manley Callin, who is preparing a check-list of the birds of the Qu'Appelle Valley. According to Mr. Callin, this is the first record for the Qu'Appelle Valley area since 1907. This brings the all-time total to 112 species seen on count days plus seven additionals seen during count period.

BANGOR, Jan. 2; 30 miles by car; temp. $-25^{\circ} ; 9$ species, 145 individuals. Great Horned Owl, 1; Downy Woodpecker, 1; Black-billed Magpie, 2; Black-capped Chickadee, 5; Bohemian Waxwing, 4; House Sparrow, 6; Pine
Grosbeak, 16; Common Redpoll, 20; Snow Bunting, 90.-Mrs. A. Thompson. BATTLEFORD, Dec. 31 ; 26 miles by car and 3 miles on foot; temp. $-15^{\circ}$; sunny and clear; 12 inches of snow; 9 species, 575 individuals. Ruffed Grouse, 2; Rock Dove, 21; Boreal Owl, 1; Horned Lark, 4; Black-billed Magpie, 1; Black-capped Chickadee, 2; Northern Shrike, 1; House Sparrow, 43; Common Redpoll, 500.Spencer G. Sealy.

BORDEN BRIDGE, Jan. 1; 22 miles in $11 / 2$ hours by car and 3 miles in 2 hours on foot; temp. $-12^{\circ}$; wind west at 6 m.p.h.; clear; about 6 inches of snow; 7 species, 106 individuals. Rough-legged Hawk, 1; Snowy Owl, 1; Downy Woodpecker, 2; Black-billed Magpie, 7; Black-capped Chickadee, 9; House Sparrow, 2; Common Redpoll, 84.-Jon, Peter and Chris Gerrard, Victor Ince, John Waddington, Doug Whitfield.

BROADVIEW, Dec. $26 ; 65$ miles by car and 2 miles on foot in 8 hours; temp. $1^{\circ}$; wind S.E. $10 \mathrm{mph}$; 9 species, 200 individuals. Mallard 3; Rock Dove, 5; Black-billed Magpie, 54 ; Black-capped Chickadee, 14 ; Bohemian Waxwing, 28; Starling, 8; House Sparrow, 53; Lapland Longspur, 25; Snow Bunting, 10.-D. G. Francis.

CHRISTOPHER LAKE, Dec. 30; about the yard all day; 5 species, 46 individuals. Blue Jay, 7; Raven, 6; Black-capped Chickadee, 12; Whitebreasted Nuthatch, 1; Evening Gros-

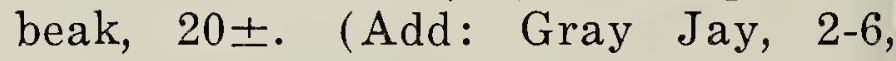
Dec. 24, 25, 29, 31, Jan. 1).-Gordon Robertson.

CRAVEN, Dec. 21: 58 miles by car and $3 \frac{1}{2}$ miles on foot in $5 \frac{1}{2}$ hours; temp. $-10^{\circ}$ to $-2^{\circ}$; no wind; clear; 2 inches of snow; 12 species, 541 individuals. Rock Dove, 3; Great Horned Owl, 3; Hairy Woodpecker, 2; Blackbilled Magpie, 32; Black - capped Chickadee, 2; Bohemian Waxwing, 87; Northern Shrike, 2; Starling, 1; 
ing, clearing in afternoon; wind SE $10 \mathrm{mph}$ in a.m.; 2 " snow; 8 species, 273 individuals. Marsh Hawk, 1; Rock Dove, 4; Short-eared Owl, 1; Horned Lark, 2; Black-billed Magpie, 12; House Sparrow, 50; Common Redpoll, 202; Snow Bunting, 1. (Add: Sharptailed Grouse, 3, Jan. 1; Gray Partridge, 7 , Dec. 22 ; Northern Shrike, 1, Dec. 30; Hawk Owl, 1, Dec. 21; Bohemian Waxwing, 8, Jan. 1).-P. Lawrence Beckie.

LADY LAKE, Dec. 28; 6 miles on foot; temp. $-5^{\circ}$; light wind; snowing lightly; 6 inches of snow; 9 species, 79 individuals. Hairy Woodpecker, 1; Downy Woodpecker, 2; Northern Three-toed Woodpecker, 2; Gray Jay, 1; Common Raven, 3; Black-capped Chickadee, 11; Boreal Chickadee, 3; Common Redpoll, 6; Snow Bunting, $50+$ - D Donald J. Buckle.

LA FLECHE, Dec. 23; 36 miles across Wood River and Pinto Creek flats; temp. $0^{\circ}$; no wind; light snow; 11 species, 199 individuals. Golden Eagle, 2; Sharp-tailed Grouse, 7; Ring-necked Pheasant, 3; Gray Partridge, 9; Rock Dove, 4; Snowy Owl, 2; Horned Lark, 100; Black-billed Magpie, 2; Starling, 3; House Sparrow, 17; Snow Bunting, 50土.-C. H. Shulver.

LEADER, Dec. 31; 24 miles by car and 3 hours afield; temp. $-10^{\circ}$; snow 5"; 4 species, 220 individuals. Sharptailed Grouse, 1; Ring-necked Pheasant, 3; Black-billed Magpie, 16; Snow Bunting, 200. (Add: Bald Eagle, 1, Dec. 27; Gray Partridge, 8, Dec. 27; Horned Lark, 2, Jan. 1).-Daisy D. Meyers.

MAPLE CREEK, Jan. 1; 25 miles by car and 1 mile on foot in 3 hours; 10 species, 246 individuals. Sharp-tailed Grouse, 9; Sage Grouse, 1; Gray Partridge, 2; Downy Woodpecker, 3; Black-billed Magpie, 11; Black-capped Chickadee, 9; Bohemian Waxwing, 3; House Sparrow, 200; Evening Grosbeak, 7; Snow Bunting, 1. (Add: 2 unidentified hawks).-Mrs. D. Bromley (compiler), John Bromley, Lloyd Burt, Brigadier Mrs. B. Hopkinson, Mrs. Stockdale, Bill Stockdale, Mrs.
E. Thomas. (Steve A. Mann Nature Club Members).

MATADOR, Dec. $20 ; 65$ miles by car and 2 miles on foot; temp. $15^{\circ}$; winds light; light snowfall; 8 species, 357 individuals. Golden Eagle, 2; Sharptailed Grouse, 5; Rock Dove, 11; Shorteared Owl, 1; Horned Lark, 182; Black-billed Magpie, 6; House Sparrow, 15; Common Redpoll, 135.-Brian Felske, Bohdan Pylypec, Alan Smith (compiler).

MOOSE JAW, Dec. 26; 75 miles by car and 15 miles on foot; temp. $2^{\circ}$; wind 15 to $19 \mathrm{mph}$; overcast with light snow; 5 inches of snow; 14 species, 1373 individuals. Gray Partridge, 13; Rock Dove, 22; Hairy Woodpecker, 2; Downy Woodpecker, 2; Horned Lark, 519; Black-billed Magpie, 25; Common Crow, 2; Blackcapped Chickadee, 15; Bohemian Waxwing, 66; Starling, 124; House Sparrow, 229; Common Redpoll, 92; Lapland Longspur, 1; Snow Bunting, 261. (Add: Ring-necked Pheasant, 2, Dec. 21 and 30; Great Horned Owl, 1, Dec. 24; Snowy Owl, 1, Dec. 22 and 27; Rusty Blackbird, 1, Dec. 30).-Ken Dodd, John Horton, Pat Kern, Leith Knight (compiler), Cy Knight, Mabel Miller, Gwen Morton, Loanne Myrha, Brian Perret, Ruth and Gordon Silversides, Craig Tufts, Ruth Way.

NIPAWIN, Dec. 26; 6 hours; temp. 0 to $-5^{\circ}$; light north wind; dense white overcast; 6" fluffy snow; 16 species, 529 individuals. Spruce Grouse, 1; Ruffed Grouse, 2; Rock Dove, 2; Great Horned Owl, 1; Hairy Woodpecker, 1 ; Downy Woodpecker, 2; Gray Jay, 2; Blue Jay, 1; Blackbilled Magpie, 10; Bohemian Waxwing, 135; Starling, 6; House Sparrow, 200; Evening Grosbeak, 75; Pine Grosbeak, 26; Hoary Redpoll, 1 ; Common Redpoll, 64. (Add: Common Goldeneye, 12, Jan. 1; Common Merganser, 3, Jan. 1; Northern Three-toed Woodpecker, 1, Dec. 28; Raven, 1, Dec. 27. Also Grea` Gray Owl, 1, Dec. 22 seen by Walter and Billie Matthews).-David and Stan Riome.

NOKOMIS, Dec. 26: 32 miles by car 
in $3^{1 / 4}$ hours; temp. $10^{\circ}$; winds light; light snow with overcast; 7 species, 455 individuals. Rock Dove, 35 ; Horned Lark, 17; Black-billed Magpie, 2; Starling, 9; House Sparrow, 30; Common Redpoll, 12; Snow Bunting, 350. (Add: Bohemian Waxwing, 7, Dec. 27).-J. W. Hamilton.

OUTLOOK, Dec. 21 ; temp. $18^{\circ}$; cloudy to clear; $2^{\prime \prime}$ of snow; 4 species, 57 individuals; Black-billed Magpie, 2; Bohemian Waxwing, 25; House Sparrow, 6; Common Redpoll, 24. (Add: Sharp-tailed Grouse, 2, Dec. 20; Gray Partridge, 9, Dec. 25; Horned Lark, 25, Dec. 25).- Harold Kvinge.

PIAPOT, Dec. $26 ; 3$ sq. mi. around farmstead; temp. $-30^{\circ}$; strong wind; 10 species, 597 individuals. Sharptailed Grouse, 70; Ring-necked Pheasant, 3; Mourning Dove, 1; Horned Lark, 321; Black-billed Magpie, 20; Black-capped Chickadee, 11; Bohemian Waxwing, 43; House Sparrow, 4; Brewer's Blackbird, 1; Tree Sparrow, 123.--Jim Benneto, Lyall Wagner.

PIKE LAKE, Dec. 28; 56 miles by car in $3 \frac{1}{2}$ hours, 2 miles on foot in 1 hour, $1 / 2$ hour at feeding station; temp. $-15^{\circ}$; wind light; steady heavy snow; 8-10 inches of snow on the level; 13 species, 1000 individuals. Ruffed Grouse, 2; Sharp-tailed Grouse, 2 ; Great Horned Owl, 1; Hairy Woodpecker, 3; Downy Woodpecker, 4; Blue Jay, 4; Black-billed Magpie, 5;

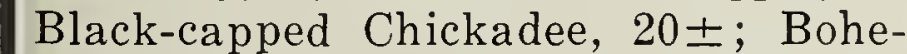
mian Waxwing, 124; House Sparrow, 2; Common Redpoll, 725; Hoary Redpoll, 2; Snow Bunting, 105. (Add: Ring-necked Pheasant, Cedar Waxwing, Northern Shrike, Evening Grosbeak).-Dr. J. B. Gollop (compiler), Mrs. J. B. Gollop, Mrs. L. M. Hoyte, F. L. Waite, J. A. Wedgwood.

PRINCE ALBERT, Dec. 22; 76 miles by car and 2 on foot in $8 \frac{1}{2}$ hours; temp. $2^{\circ}$ to $13^{\circ}$; wind $\mathrm{N} 14 \mathrm{mph}$; 4 inches of snow in open, 6 inches in the woods; 17 species, 491 individuals. Rock Dove, 1; Hawk Owl, 1; Hairy Woodpecker, 2; Downy Woodpecker, 2; Northern Three-toed Woodpecker,
1; Gray Jay, 2; Blue Jay, 1; Blackbilled Magpie, 6; Common Raven, 40; Black-capped Chickadee, 13; Boreal Chickadee, 3; Bohemian Waxwing, 8; House Sparrow, 37; Evening Grosbeak, 82; Pine Grosbeak, 32; Hoary Redpoll, 6; Common Redpoll, 254.Mr. and Mrs. O. A. Aschim, Elizabeth Beacham and E. Derek Beacham (compiler).

RADISSON, Jan. $1 ; 1$ 1/2 miles on foot in 1 hour; temp. $2^{\circ}$; $6^{\prime \prime}$ of snow; 5 species, 1018 individuals. Hairy Woodpecker, 1; Black-billed Magpie, 11; Black-capped Chickadee, 6; House Sparrow, 700; Snow Bunting, 300. (Add: Sharp-tailed Grouse, 7, Dec. 20; Barred Owl, 1, Dec. 20 and 27; Long-eared Owl, 1, Dec. 20).-Bryan Rothenburger.

RAYMORE, Dec. 21; 100 miles by car; temp. $10^{\circ}$; wind SE $15 \mathrm{mph}$; 8 species, 328 individuals. Ruffed Grouse, 1; Rock Dove, 6; Black-billed Magpie, 4; Black-capped Chickadee, 4; Northern Shrike, 1; House Sparrow, 75; Pine Grosbeak, 7; Snow Bunting, 230. (Add: Great Horned Owl, 3, Dec. 24 and Jan. 1; Hairy Woodpecker, 1, Jan. 1; Bohemian W a xwing, 2, Dec. 22). - Wayne Harris.

REGINA, Dec. 28; 44 miles on foot in 9 hours, and 203 miles by car in 9 hours; temp. $-13^{\circ}$ to $-9^{\circ}$; blizzard conditions; wind NW $30 \mathrm{mph}$; 4 inches snow cover; 26 species, 3618 individuals. Red-necked Grebe, 1; Pied-billed Grebe, 1; Mute Swan, 10; Whistling Swan, 4; Canada Goose, 454; Mallard, 230; Common Goldeneye, 1 ; Sharp-tailed Grouse, 1; American Coot, 2; Rock Dove, 29; Great Horned Owl, 1; Snowy Owl, 1; Shorteared Owl, 1; Hairy Woodpecker, 2; Downy Woodpecker, 4; Horned Lark, 88; Black-billed Magpie, 41; Blackcapped Chickadee, 19; Red-breasted Nuthatch, 1; Bohemian Waxwing, 230; Northern Shrike, 2; Starling, 17; House Sparrow, 1812; Common Redpoll, 198; Slate-colored Junco, 1; Snow Bunting, 467. (Add: Gadwall, 1, Dec. 20; Rough-legged Hawk, 1, Jan. 1; Gray Partridge, 15, Dec. 26; 
Yellow-shafted Flicker, 1, Dec. 20; Brown Thrasher, 1, Dec. 21; Redwinged Blackbird, 3, Dec. 23; Pine Grosbeak, Dec. 26; Purple Finch, 1, Dec. 20.) - Gary Anweiler, Jessie Bailey, Margaret Belcher, Betty Binnie, Greg Bobbitt, Frank Brazier, Bill Brownlee, Eric Cooke, Bill Eddie, Doug Gilroy, Jay Jordan, George Ledingham, Helen Morrison, Mr. and Mrs. J. F. McKay, Marg Patterson, Connie Pratt, Joe Roberts, Barbara Ross, Lorne Scott, Dr. Smith, Doug Smith, Jim Taylor, Ruth Tempel, Holly Wallace, Janie Wilhelm, Dick Yeaton. (Al and Betty Binnie, compilers.)

SALTCOATS, Dec. 26; 10 miles by car and 6 miles on foot in 8 hours: temp. $-5^{\circ}$ to $10^{\circ}$; wind SE $10 \mathrm{mph}$; intermittent light snow; 7 species, 113 individuals. Ruffed Grouse, 1; Downy Woodpecker, 1; Black-capped Chickadee, 5; Bohemian Waxwing, 50; Pine Grosbeak, 12; House Sparrow, 35; Black-billed Magpie, 9. (Add: Sharp-tailed Grouse, 5; Gray Partridge, 7; Great Gray Owl, 1; Hairy Woodpecker, 1).- Harold Jowsey, Harriet Jowsey, J. Norman Jowsey, J..R. Jowsey (compiler), Eric Upshal.

SASKATOON, Dec. 26; 35 miles on foot in 27 hours, 246 miles by car in 30 hours, 20 miles by ski-doo in 4 hours; temp. $-3^{\circ}$ to $-7^{\circ}$; wind NW 7 to $10 \mathrm{mph}$; overcast with snow in the a.m.; clearing briefly in the p.m.; 36 species, 6858 individuals. Mallard, 41; Gadwall, 1; American Widgeon, 1; Goshawk, 1; Rough-legged Hawk, 1; Golden Eagle, 1; Pigeon Hawk, 1; Ruffed Grouse, 3; Sharp-tailed Grouse, 138; Ring-necked Pheasant, 15; Gray Partridge, 183; Rock Dove, 130; Great Horned Owl, 2; Short-eared Owl, 13; Yellow - shafted Flicker, 1; Hairy Woodpecker, 12; Downy Woodpecker, 5; Horned Lark, 90; Blue Jay, 13; Black-billed Magpie, 169; Blackcapped Chickadee, 48; White-breasted Nuthatch, 1; Red-breasted Nuthatch, 1; Robin, 3; Bohemian Waxwing, 2846; Cedar Waxwing, 6; Northern Srike, 1; Starling, 6; House Sparrow, 1493; Rusty Blackbird, 1; Evening Gros- beak, 17; Pine Grosbeak, 23; Hoary Redpoll, 17; Common Redpoll, 1492; Slate-colored Junco, 2; Snow Bunting, 81. (Add: Snowy Owl, 1, Dec. 22, 31; Red-winged Blackbird, 10, Dec. 28).Saskatoon Natural History Society: Claude Anderson, Rod and Michael Bantjes, Bob, Jeff and Joan Besant, Edward and Ray Bisha, Randy and Vern Bowman, Wm. Bruchs, John Cartwright, Pern Cordery, Mrs. Molly Denson, Annabel and Louise Denson, Mrs. Rosalie Early, Ed Ellaschuk, Bill and Kim Epp, Claire and Julie Ewing, L., Danny and Gerald Fitzgerald, R. Flint, Jon and Peter Gerrard, Michael A. Gollop, Dr. and Mrs. J. B. Gollop, David V. Houston, Dr. and Mrs. C. Stuart Houston, Dr. A. Lambert, Kim and Ronald A. Lamont, Dennis McBride, Greg Michalenko, Allan Moulain, Mrs. J. B. Nicholson, Arnold Nijssen, David Nowasad, Bohdan Pylypec, Ron Shore, Stanley J. Shadick, Jas. A. Slimmon, Alan R. Smith, Mr. and Mrs. Thos. R. Smith, Fred Waite, Jas. A. Wedgwood. SKULL CREEK, Dec. 27; 2 miles on foot down the creek; temp. $-25^{\circ} ; 10$ species, 126 individuals. Sharp-tailed Grouse, 9; Ring-necked Pheasant, 2; Gray Partridge, 7; Hairy Woodpecker, 4; Downy Woodpecker, 2; Horned Lark, 28; Black-billed Magpie, 9; Black-capped Chickadee, 12; Bohemian Waxwing, 20; House Sparrow, 23. (Add: Golden Eagle, 2, Dec. 21; Snowy Owl, 1, Dec. 21; Common Redpoll, 20, Dec. 23; Snow Bunting, 1, Dec. 21).-Mrs. S. A. Mann (compiler), Robert and Betty Mann, Patti, Jim and Dougie Mann, Helen, Ray, Michelle and Tommy Schuler, Peter Swain.

SPIRIT LAKE, Dec. 26; 2 miles on foot through poplar and spruce bush, and around yard in 4 hours; temp. $0^{\circ}$; cloudy with clear periods; 8" snow; snowflurries; wind light; 14 species, 73 individuals. Ruffed Grouse, 3; Great Horned Owl, 2; Pileated Woodpecker, 1; Hairy Woodpecker, 4; Downy Woodpecker, 6; Blackbilled Magpie, 1; B lack-cap ped Chickadee, 30: White-breasted Nuthatch, 2; Bohemian Waxwing, 8; 
Northern Shrike, 1; Evening Grosbeak, 5; Pine Grosbeak, 3; Common Redpoll, 5; Snow Bunting, 2. (Add: Robin, Dec. 21).-Joyce Gunn, Mrs. Gunn.

TUBEROSE, Dec. $26 ; 161 / 2$ miles by car and $1 / 2$ mile on foot; temp. $-20^{\circ}$; wind NW $10 \mathrm{mph}$; visibility good; 6 " of snow; 6 species, 66 individuals. Pigeon Hawk, 1; Gray Partridge, 39; Black-billed Magpie, 1; Bohemian Waxwing, 3; House Sparrow, 7 ; Common Redpoll, 15.-C. A. Matthews.

VAL MARIE, Dec. 21; 8" snow; 5 species, 12 individuals. Golden Eagle, 1; Sharp-tailed Grouse, 2; Horned Lark, 5; Starling, 1; Snow Bunting, 3. (Add: Rough-legged Hawk, 1, Dec. 20 ; Unidentified Falcon, 1, Dec. 25; Ring-necked Pheasant, 10, Dec. 31; Gray Partridge, 5, Dec. 22; Shorteared Owl, 1, Dec. 20; Black-billed Magpie, 2, Dec. 20 ; Black-capped Chickadee, 1, Dec. 31; Bohemian Waxwing, 1, Dec. 26; House Sparrow, 12, Dec. 22; Red-winged Blackbird, 1, Dec. 25; Common Redpoll, 6, Dec. 31; Tree Sparrow, 1, Dec. 25).-J. David Chandler.

WOLSELEY, Dec. $31 ; 20$ miles by car; temp. $-10^{\circ}$; clear; 5 inches of snow; 10 species, 84 individuals. Ruffed Grouse, 2; Great Horned Owl, 1; Hairy Woodpecker, 1; Downy Woodpecker, 2; Black-billed Magpie, 2; Black-capped Chickadee, 4; Starling, 1; House Sparrow, 50; Common Redpoll, 20; Snow Bunting, 1. (Add: Short-eared Owl, 2, Dec. 23; Horned Lark, 20, Dec. 27; Bohemian Waxwing, 16, Dec. 28).-J. Donald Hayward.

YELLOW CREEK, Dec. 30; 6 miles on foot for 6 hours; temp. $-28^{\circ}$ to $-15^{\circ}$; wind W $15 \mathrm{mph}$; overcast with light snow; 24 inches of snow; 6 species, 191 individuals. Downy Woodpecker, 1; Blue Jay, 3; Blackbilled Magpie, 5; Black-capped Chickadee, 2; Pine Grosbeak, 57; Common Redpoll, 61.-Derwent Mazur.

YORKTON, Dec. $26 ; 85$ miles by car and 13 miles on foot for 42 hours; temp. $1^{\circ}$ to $4^{\circ}$; wind SSE $10 \mathrm{mph}$ dropping in p.m.; 1 inch snowfall during the day; 4 inches of snow; 15 species, 2895 individuals. Sharp-tailed Grouse, 30; Gray Partridge, 1; Rock Dove, 37; Great Horned Owl, 1; Snowy Owl, 2; Downy Woodpecker, 2; Horned Lark, 1; Black-billed Magpie, 37; Black-capped Chickadee, 22; Bohemian Waxwing, 106; Starling, 24; House Sparrow, 258; Pine Grosbeak, 2; Common Redpoll, 870; Snow Bunting, 1500.-Wm. Anaka, Lorne Lepp, Larry Morgotch, Irving and Gordon Pearce, Wm. Popowich, Frank Switzer (compiler), Harold Wilkinson (Yorkton Senior Natural History Society); and Keith Monette (Yorkton Junior Natural History Society). EDMONTON, ALTA. Dec. $22 ; 39$ miles on foot in $21 \frac{1 / 4}{4}$ hours, and 203 miles by car in $22 \%$ hours; temp. $1^{\circ}$ to $-11^{\circ}$; clear; wind $\mathrm{NW} 4 \mathrm{mph} ; 6$ inches of snow; 29 species, 6,131 individuals. Mallard, 42; Common Goldeneye, 18; Pigeon Hawk, 1; Ruffed Grouse, 1; Ring-necked Pheasant, 15; Gray Partridge, 96; Rock Dove, 70; Great Horned Owl, 1; Snowy Owl, 1; Hawk Owl, 1; Short-eared Owl, 4; Yellow-shafted Flicker, 1; Pileated Woodpecker, 2; Hairy Woodpecker, 7; Downy Woodpecker, 6; Black-backed Three-toed Woodpecker, 1; Blue Jay, 30; Black-billed Magpie, 119; Blackcapped Chickadee, 82; Golden-crowned Kinglet, 1 ; $\mathrm{B}$ o hemia $\mathrm{n}$ Waxwing, 5,000; Northern Shrike, 1; Starling, 16; House Sparrow, 305; Evening Grosbeak, 19; Pine Grosbeak, 10; Hoary Redpoll, 2; Common Redpoll, 179; Snow Bunting, 100. (Add: Pintail, Golden Eagle).-A. Allan, T. Balacko, K. Ball, H. Campbell, P. Demulder, R. Heath, M. Hennie, O. Hohn, H. Horton, B. Horton, R. Lister, A. MacGregor, J. Park, D. Robinson, H. Smith, D. Spalding, P. Taylor, P. Thompson, R. Turner (compiler).

FORT SMITH, N.W.T., Dec. $29 ; 40$ miles by car in 2 hours; temp. $-17^{\circ}$; wind $\mathrm{N} 15 \mathrm{mph}$; overcast; 12 inches of snow; 4 species, 38 individuals. Willow Ptarmigan, 4; Rock Dove, 7; Raven 16; Hoary Redpoll, 11. (Add: House Sparrow, 6, Dec. 27; Pine Grosbeak, 20, Dec. 27).-Elsie, Ernie and Pamela Kuyt. 